\title{
Tecnura
}

\section{Diseño y construcción de una interfaz háptica de seis grados de libertad}

\section{Design and construction of a 6 dlof haptic device}

\author{
Yamid Estiven Otaya Bravo1, Marco Fidel Piamba Mamián², Oscar Andrés Vivas Albán³
}

Fecha de recepción: 2 de marzo de 2015

Fecha de aceptación: 28 de agosto de 2017

Cómo citar: Otaya B., Y.E.; Piamba M., M.F. y Vivas A., O.A. (2017). Diseño y construcción de una interfaz háptica de seis grados de libertad. Tecnura, 21(54), 33-40. https://doi.org/10.14483/22487638.8229

\section{RESUMEN}

Contexto: Múltiples aplicaciones tecnológicas están empezando a incluir dispositivos de realimentación háptica, los cuales envían al usuario algún tipo de realimentación que a su vez se traduce en sensación. Este trabajo incursiona en la construcción de uno de estos dispositivos hápticos.

Método: El presente trabajo muestra el procedimiento de diseño y posterior construcción de una interfaz háptica que permite seis grados de movimiento independiente con su respectiva realimentación.

Resultados: Se obtuvo una interfaz háptica de seis grados de libertad, la cual permite el movimiento de un cursor en un ambiente virtual constituido por un cubo dentro del computador. Dicha interfaz envía al usuario una realimentación de fuerza cuando el cursor virtual toca alguno de los lados del cubo virtual. Conclusiones: La interfaz háptica construida proporciona una adecuada sensación háptica en el ambiente virtual construido. Dicha interfaz podrá ser probada y utilizada en diversos proyectos que pretendan ofrecer mayor percepción al usuario.

Palabras clave: Interfaces hápticas, Robótica, Simulación virtual.

\section{ABSTRACT}

Context: Many technological applications are including haptic devices, which send to the user haptic feedback that is traduced to a sensation. This work shows the construction of a type of these devices.

Method: This work shows the design and construction of a haptic device of six degrees of independent movement with its force feedback.

Results: It was obtained a haptic device of six degrees of freedom that allows the movement of a cursor on a virtual environment formed by a cube in the PC. The haptic device sends to the user a force feedback when the cursor touches any side of the virtual cube.

Conclusions: The built haptic device provides a suitable haptic sensation into the virtual environment. This device will be able to test and use in many advanced perception projects.

Keywords: Haptic devices, Robotics, Virtual simulation.

\footnotetext{
Ingeniero en Automática Industrial. Universidad del Cauca. Popayán, Colombia. Contacto: estiven805@hotmail.com Ingeniero en Automática Industrial, Universidad del Cauca. Popayán, Colombia. Contacto: marcopiamba1@gmail.com Doctor en Robótica, Universidad del Cauca. Popayán, Colombia. Contacto: avivas@unicauca.edu.co
} 


\section{INTRODUCCIÓN}

Lo últimos años han brindado un gran desarrollo tecnológico en aquellos dispositivos llamados hápticos, que son diversos mecanismos capaces de ofrecer al usuario una realimentación de fuerza proveniente de un mundo virtual. Estos mecanismos permiten tocar, sentir, manipular o cambiar objetos que están presentes en un ambiente virtual representado en el computador, añadiendo el sentido del tacto a la experiencia visual. En otras palabras, el usuario no solamente envía datos al computador, sino que recibe información proveniente de este, a través del mecanismo háptico, el cual le genera una sensación física en alguna parte de su cuerpo. Si la sensación física se percibe en las manos se realiza por medio de un guante, si es en sus extremidades será a través de un exoesqueleto. O puede simplemente realimentar la fuerza al usuario por medio de un dispositivo de entrada, similar a un joystick. Actualmente existen muchos dispositivos comerciales, algunos dedicados a los videojuegos, y otros más avanzados utilizados en diversos campos de investigación, como los populares Phantom de Sensable (Salisbury y Srinivasan, 1997), Butterfly Haptics (Hollis, 2010) o HapticMaster (Van der Linde, Lammertse, Frederiksen y Ruiter, 2002), entre otros. Un resumen de este tipo de dispositivos puede encontrarse en Tiwana, Redmond y Lovell (2012).

Las aplicaciones de las interfaces hápticas son numerosas, y van desde la simulación en la industria aeroespacial hasta su incursión en el diagnóstico y práctica quirúrgica (Vander Poorten, Demeester y Lammertse, 2012; Abate et al., 2009). Se observa, entonces, la importancia de disponer de un dispositivo háptico tipo joystick como apoyo tecnológico a las numerosas tareas que abarca la investigación hoy en día (Ohnishi, Shimono y Natori, 2009). La Universidad del Cauca, en Colombia, desarrolla actualmente diversos proyectos en el área de la simulación médica (Salinas y Vivas, 2009; Mosquera, Vivas y Rengifo, 2011; Vivas,
Rengifo y Mosquera, 2011; Vera y Vivas, 2013), muchos de los cuales requieren de dispositivos hápticos para una mejor comprensión de los procesos que involucran. Por tal motivo se decidió construir una interfaz háptica propia, de seis grados de libertad. En el presente artículo se muestra el diseño utilizado y la posterior construcción de dicha interfaz, la cual fue denominada Haptic 6DOF.

\section{METODOLOGÍA}

\section{Modelado de la interfaz háptica}

Con el fin de que la interfaz Haptic $6 D O F$ no solo brindara la posibilidad de ubicar un objeto virtual en un espacio de tres dimensiones, sino que también le imprimiera rotación en cualquiera de sus tres ejes, se escogió una estructura de seis grados de libertad similar a la del popular robot Puma. La figura 1 muestra la arquitectura de la interfaz diseñada.

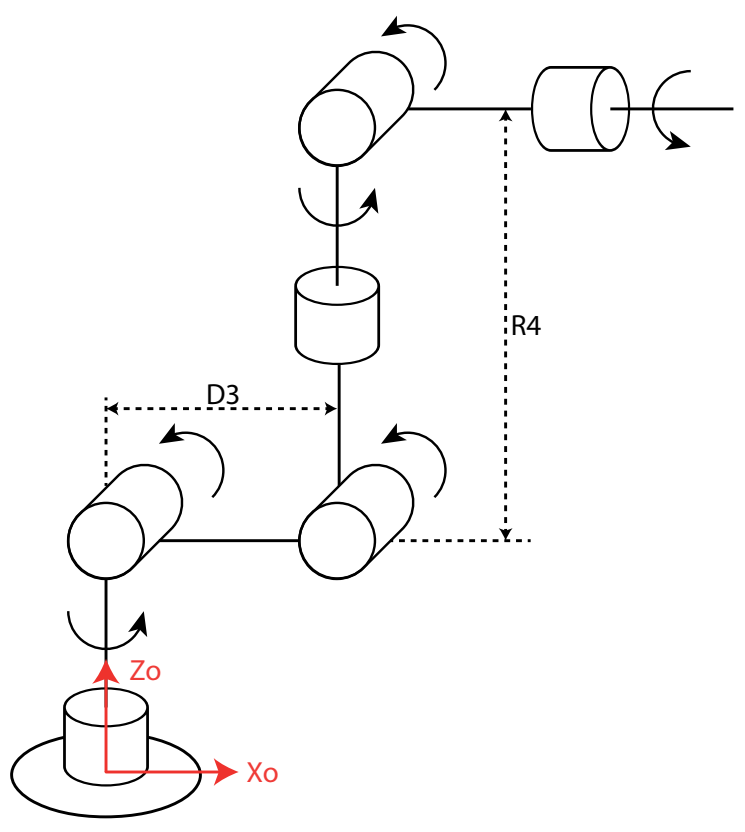

Figura 1. Estructura cinemática de la interfaz Haptic $6 D O F$

Fuente: elaboración propia. 
Siguiendo la metodología de Khalil-Kleinfinger (1986) se obtuvo la tabla 1, correspondiente a los parámetros geométricos de la interfaz.

Tabla 1. Parámetros geométricos de la interfaz Haptic $6 D O F$

\begin{tabular}{cccccc}
\hline $\boldsymbol{j}$ & $\boldsymbol{\sigma j}$ & $\boldsymbol{\alpha j}$ & $\boldsymbol{d j}$ & $\boldsymbol{\theta j}$ & $\boldsymbol{r j}$ \\
\hline 1 & 0 & 0 & 0 & $\theta 1$ & 0 \\
\hline 2 & 0 & 90 & 0 & $\theta 2$ & 0 \\
\hline 3 & 0 & 0 & $\mathrm{D} 3$ & $\theta 3$ & 0 \\
\hline 4 & 0 & -90 & 0 & $\theta 4$ & $\mathrm{R} 4$ \\
\hline 5 & 0 & 90 & 0 & $\theta 5$ & 0 \\
\hline 6 & 0 & -90 & 0 & $\theta 6$ & 0 \\
\hline
\end{tabular}

Fuente: elaboración propia.

Donde:

j representa el número de la articulación o cuerpo.

бj indica si la articulación es de traslación (1) o de rotación (0).

aj y $\theta j$ son ángulos que dependen de los ejes de las articulaciones o de la rotación de una articulación rotacional.

dj y rj son distancias entre los ejes de las articulaciones o que involucran el desplazamiento de una articulación prismática.

A partir de dicha tabla se hallan tanto el modelo geométrico directo, como los modelos dinámicos necesarios para la simulación y el control de la interfaz (Vaca, Pe-a y Vacca, 2015). El modelo geométrico directo permite conocer la posición y orientación del órgano terminal de la interfaz, en función de las posiciones de cada una de sus seis articulaciones. Se define por la matriz ${ }^{0} \boldsymbol{T}_{6}$, matriz 4×4 (ecuación (1)).

$$
{ }^{i} \boldsymbol{T}_{j}=\left[\begin{array}{llll}
{ }^{i} \boldsymbol{s}_{j} & { }^{i} \boldsymbol{n}_{j} & { }^{i} \boldsymbol{a}_{j} & { }^{i} \boldsymbol{P}_{j}
\end{array}\right]=\left[\begin{array}{cccc}
s_{x} & n_{x} & a_{x} & P_{x} \\
s_{y} & n_{y} & a_{y} & P_{y} \\
s_{z} & n_{z} & a_{z} & P_{z} \\
0 & 0 & 0 & 1
\end{array}\right]
$$

Donde cada expresión de la matriz anterior equivale a lo detallado en la tabla 2.
Tabla 2. Valores de los términos de la matriz ${ }^{0} \boldsymbol{T}_{6}$

\begin{tabular}{l}
\hline$s_{x}=\mathrm{C} 1(\mathrm{C} 23(\mathrm{C} 4 \mathrm{C} 5 \mathrm{C} 6-\mathrm{S} 4 \mathrm{~S} 6)-\mathrm{S} 23 \mathrm{~S} 5 \mathrm{C} 6)-\mathrm{S} 1(\mathrm{~S} 4 \mathrm{C} 5 \mathrm{C} 6+\mathrm{C} 4 \mathrm{~S} 6)$ \\
$S_{y}=\mathrm{S} 1(\mathrm{C} 23(\mathrm{C} 4 \mathrm{C} 5 \mathrm{C} 6-\mathrm{S} 4 \mathrm{~S} 6)-\mathrm{S} 23 \mathrm{~S} 5 \mathrm{C} 6)+\mathrm{C} 1(\mathrm{~S} 4 \mathrm{C} 5 \mathrm{C} 6+\mathrm{C} 4 \mathrm{~S} 6)$ \\
$s_{z}=\mathrm{S} 23(\mathrm{C} 4 \mathrm{C} 5 \mathrm{C} 6-\mathrm{S} 4 \mathrm{~S} 6)+\mathrm{C} 23 \mathrm{~S} 5 \mathrm{C} 6$ \\
\hline$n_{x}=\mathrm{C} 1(-\mathrm{C} 23(\mathrm{C} 4 \mathrm{C} 5 \mathrm{~S} 6-\mathrm{S} 4 \mathrm{C} 6)+\mathrm{S} 23 \mathrm{~S} 5 \mathrm{~S} 6)+\mathrm{S} 1(\mathrm{~S} 4 \mathrm{C} 5 \mathrm{~S} 6+\mathrm{C} 4 \mathrm{C} 6)$ \\
\hline$n_{y}=\mathrm{S} 1(-\mathrm{C} 23(\mathrm{C} 4 \mathrm{C} 5 \mathrm{~S} 6-\mathrm{S} 4 \mathrm{C} 6)+\mathrm{S} 23 \mathrm{~S} 5 \mathrm{~S} 6)-\mathrm{C} 1(\mathrm{~S} 4 \mathrm{C} 5 \mathrm{~S} 6+\mathrm{C} 4 \mathrm{C} 6)$ \\
\hline$n_{z}=-\mathrm{S} 23(\mathrm{C} 4 \mathrm{C} 5 \mathrm{~S} 6+\mathrm{S} 4 \mathrm{C} 6)-\mathrm{C} 23 \mathrm{~S} 5 \mathrm{~S} 6$ \\
$a_{x}=-\mathrm{C} 1(\mathrm{C} 23 \mathrm{C} 4 \mathrm{~S} 5+\mathrm{S} 23 \mathrm{C} 5)+\mathrm{S} 1 \mathrm{~S} 4 \mathrm{~S} 5$ \\
$a_{y}=-\mathrm{S} 1(\mathrm{C} 23 \mathrm{C} 4 \mathrm{~S} 5+\mathrm{S} 23 \mathrm{C} 5)-\mathrm{C} 1 \mathrm{~S} 4 \mathrm{~S} 5$ \\
$a_{z}=-\mathrm{S} 23 \mathrm{C} 4 \mathrm{~S} 5+\mathrm{C} 23 \mathrm{C} 5$ \\
$P_{x}=-\mathrm{C} 1(\mathrm{~S} 23 \mathrm{R} 4-\mathrm{C} 2 \mathrm{D} 3)$ \\
\hline$P_{y}=-\mathrm{S} 1(\mathrm{~S} 23 \mathrm{R} 4-\mathrm{C} 2 \mathrm{D} 3)$ \\
\hline$P_{z}=\mathrm{C} 23 \mathrm{R} 4+\mathrm{S} 2 \mathrm{D} 3$
\end{tabular}

Fuente: elaboración propia.

Donde, por ejemplo, $\mathrm{C} 1=\cos (\theta 1), \mathrm{S} 23=\sin (\theta 2+\theta 3)$, etc.

En el caso de la interfaz háptica, el modelo geométrico directo es primordial ya que permite mover el cursor virtual en un espacio de tres dimensiones según los valores articulares medidos en cada articulación de la interfaz. Es decir, el usuario, al mover la interfaz háptica, se mueven inicialmente de manera libre los motores que la conforman; dicho movimiento es captado por los potenciómetros situados en cada motor, traduciéndose finalmente en un movimiento cartesiano en el ambiente virtual. Mayores detalles sobre este modelo pueden consultarse en Vivas (2010).

Para hallar los modelos dinámicos (el modelo dinámico directo es necesario para simular el comportamiento de la interfaz; el modelo dinámico inverso para la implementación de un controlador basado en el modelo), se utilizó el software Symoro (Khalil y Creusot, 1997). Este software entrega simbólicamente las ecuaciones de estos modelos para posteriormente realizar la simulación en el ambiente Matlab-Simulink $®$. La figura 2 muestra el esquema de control por par, calculado e implementado, con el fin de probar el modelo matemático de la interfaz Haptic 6DOF (Bueno, Arteaga y Candea, 2013). 


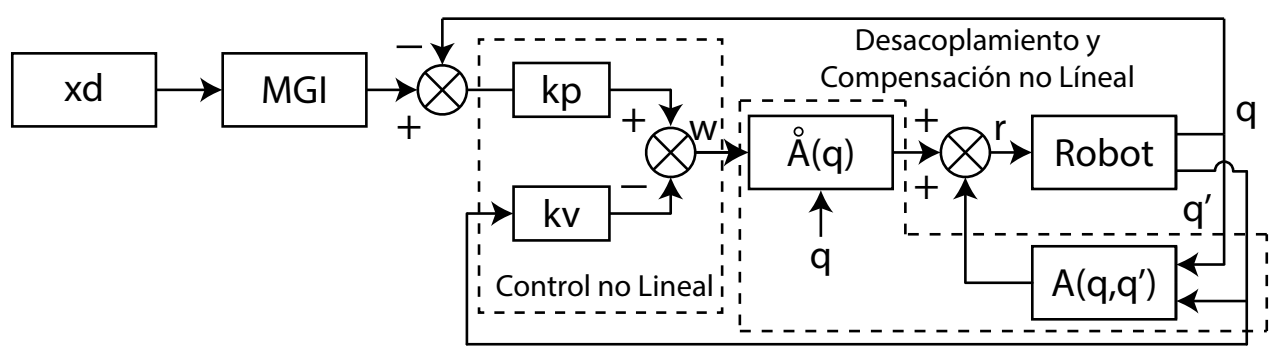

Figura 2. Control por par calculado aplicado al modelo de la interfaz propuesta

Fuente: elaboración propia.

La simulación permite validar el modelo matemático propuesto y, lo más importante, obtener los pares necesarios para los seis motores que tendrá la interfaz. Los valores de los parámetros dinámicos de la interfaz (masas e inercias) fueron obtenidos a partir del software Solid Edge®. Los resultados en simulación arrojaron que los motores necesarios, como máximo, deben proveer un par de 0,23 N.m, a partir de este dato se procedió a la adquisición tanto de ellos como de la electrónica adicional necesaria.

\section{Construcción de la interfaz háptica}

Gracias al acercamiento del modelo físico de la interfaz Haptic 6DOF obtenido por medio del software SolidEdge ${ }^{\circledR}$ se determinó que los materiales adecuados para la construcción de la interfaz son el polipropileno (acrílico) y el aluminio para la construcción de las articulaciones, y el Mdf para la base de la interfaz.

Como actuador principal se utilizó el servo digital Herkulex DRS 0101 y como actuador secundario el servo análogo H-King 15178, los cuales se ajustan a los requerimientos que surgieron de la fase de simulación. Como sensor, se manejó un potenciómetro logarítmico, que de acuerdo con el tipo de articulación en el que se ubica, recibe el nombre de sensor secundario para las articulaciones de orientación y sensor de posición para las articulaciones de posición. Además, usó una tarjeta Arduino Mega 2560 para la comunicación entre el computador y el hardware de la interfaz, así como de una fuente de alimentación y un regulador de voltaje.

La realimentación háptica hacia el usuario se obtiene por medio de un conjunto de resortes y su actuador, los cuales generan la fuerza contraria necesaria para producir el efecto de realimentación. Esta fuerza se obtiene ya que existen dos placas paralelas, una metálica y otra acrílica, las cuales se unen por medio de dos resortes. El conjunto de resortes, placas, actuador y sensor puede observarse en la figura 3.

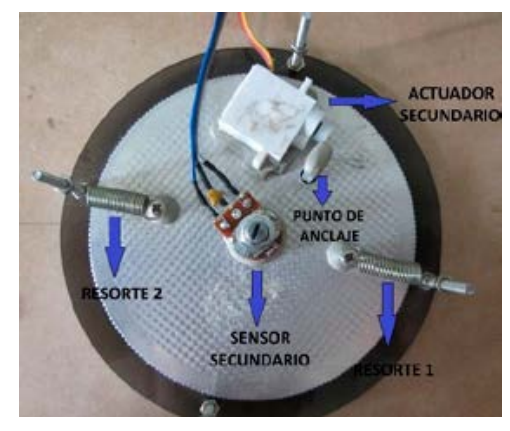

Figura 3. Componentes del sistema de realimentación Fuente: elaboración propia.

Cuando no existe ningún evento de colisión virtual, las dos placas se mueven conjuntamente debido a la fuerza que ejerce el usuario. Pero en el momento en que se presenta una colisión virtual, el actuador secundario desancla las placas, lo que genera una fuerza de restitución contraria a 
la fuerza ejercida por el usuario. Esta dirección de fuerzas se muestra en la figura 4.

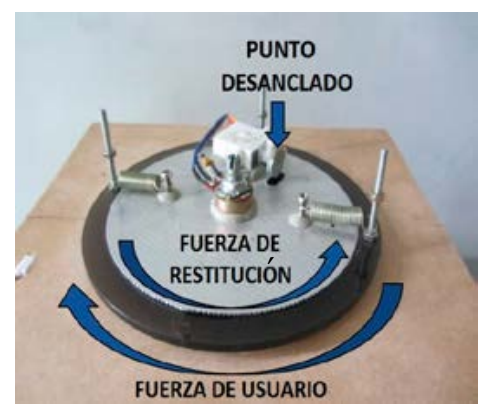

Figura 4. Dirección de las fuerzas que actúan cuando hay colisión virtual

Fuente: elaboración propia.

Finalmente, la figura 5 muestra las tres primeras articulaciones de la interfaz, las cuales proveen el posicionamiento de esta, cada una con un mecanismo basado en placas como el que acaba de explicarse.

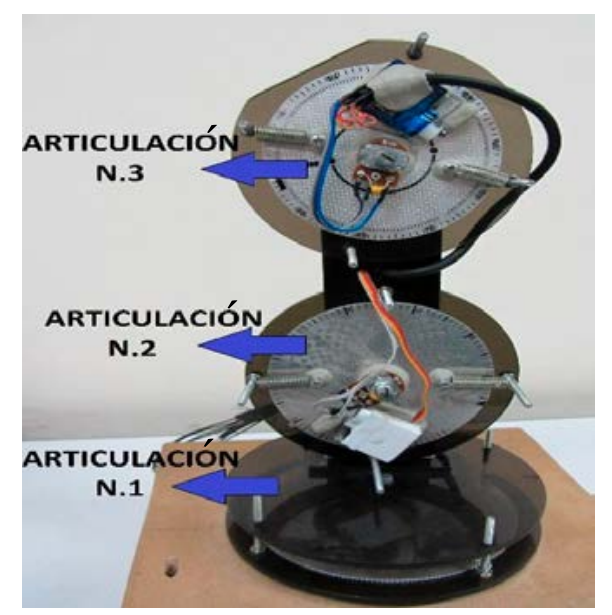

Figura 5. Articulaciones de posicionamiento y su mecanismo de realimentación

Fuente: elaboración propia.

Para las tres últimas articulaciones, el caso es más sencillo puesto que no proveen realimentación alguna. Ellas están constituidas por piezas cilíndricas de PVC que contienen los sensores de orientación que permitirán girar el puntero virtual. La figura 6 muestra estas tres articulaciones.

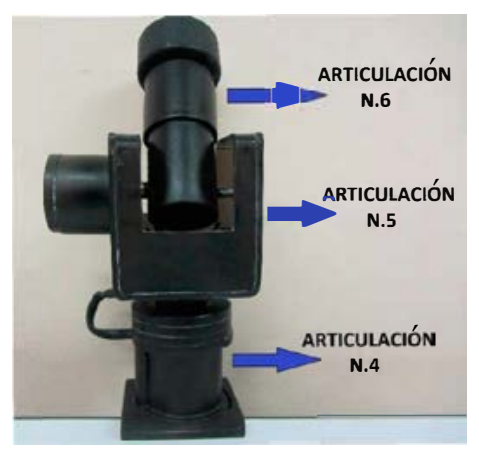

Figura 6. Articulaciones de orientación

Fuente: elaboración propia.

A continuación, se explica con mayor detalle el proceso de realimentación:

- Primer evento. Inicialmente, dado que no se requiere realimentación de fuerzas, las placas de aluminio y acrílico se mueven conjuntamente gracias a que el actuador secundario las mantiene ancladas.

- Segundo evento. Una vez el usuario ha tocado una zona de colisión virtual, el actuador secundario desancla las placas, permitiendo que el actuador principal que se encuentra unido con la placa de aluminio ejerza una fuerza de restitución contraria a la ejercida por el usuario. Una vez se presenta este evento, se pueden presentar tres tipos de realimentación: suave (XS), la cual se simula mediante un ángulo de giro de 10 grados realizados por el actuador principal y contrario a la fuerza ejercida por el usuario; media (XM), simulada por un ángulo de giro de 20 grados; y fuerte (XF), simulada por un ángulo de giro de 30 grados.

\section{Software para el control de la interfaz}

El software de control se utiliza para el manejo de la Haptic 6DOF en el control de sus dispositivos electrónicos (actuadores y sensores), además de permitir la comunicación de tipo serial entre 
la tarjeta de control Arduino y el computador del usuario. La tarjeta Arduino incluye librerías que permiten la comunicación directa con los servomotores digitales que utiliza el dispositivo. También, trae librerías para la conexión de los demás actuadores y sensores analógicos.

Para la construcción de la interfaz de usuario se usó la plataforma Visual Studio, además de los programas y bibliotecas de software libre Qt, Blender, VTK y Cmake. Para la detección de colisiones se utilizaron las librerías $V$-Collide, desarrolladas para trabajar en ambientes que contienen figuras geométricas formadas por mallas (triángulos), conservando información acerca de dónde se encuentran los objetos en el ambiente. El proceso de implementación de este algoritmo se basa en el reconocimiento del punto de colisión en la superficie del objeto inmóvil, en este caso una figura geométrica simple (cubo) (Gómez y Flórez, 2016). La función se utiliza para activar la fuerza de realimentación del dispositivo háptico, reconociendo a la vez la respectiva ID del punto de colisión. La figura 7 muestra el entorno inicial y la definición de los puntos de colisión.

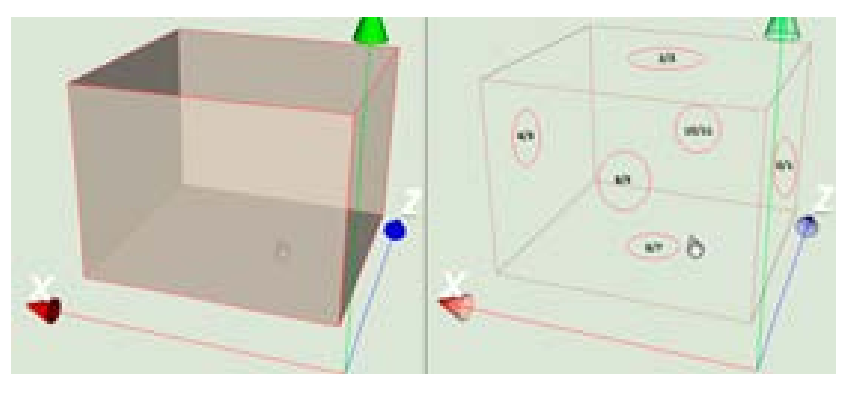

Figura 7. Definición de los puntos de colisión

Fuente: elaboración propia.

\section{RESULTADOS}

Las siguientes figuras muestran las pruebas finales obtenidas con la interfaz Haptic 6DOF: movimiento del cursor virtual sobre el eje $X$ sin contacto con las paredes del cubo (figuras 8 y 9); contacto con la parte superior del cubo en el eje $Y$, produciendo una realimentación media (figura 10); y variación de la orientación sobre el eje Z (figuras 11 y 12).

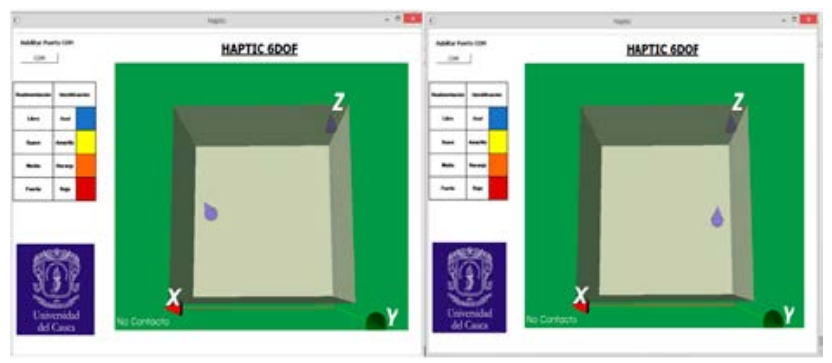

Figura 8. Variación de la posición en $X$ del cursor virtual Fuente: elaboración propia.
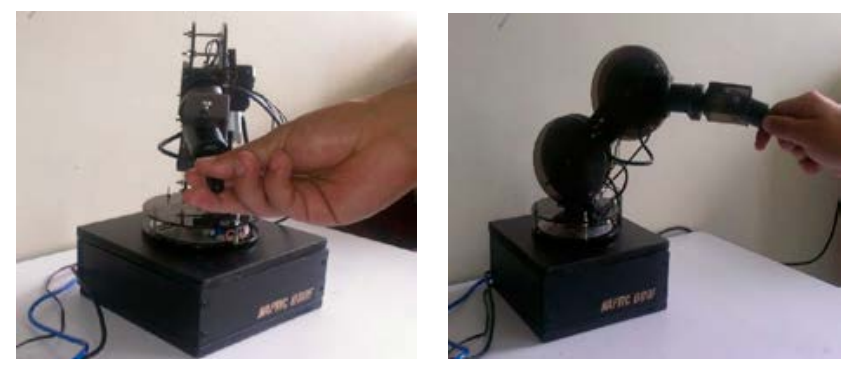

Figura 9. Movimiento de la interfaz mientras se varía la posición en $X$ del cursor virtual

Fuente: elaboración propia.

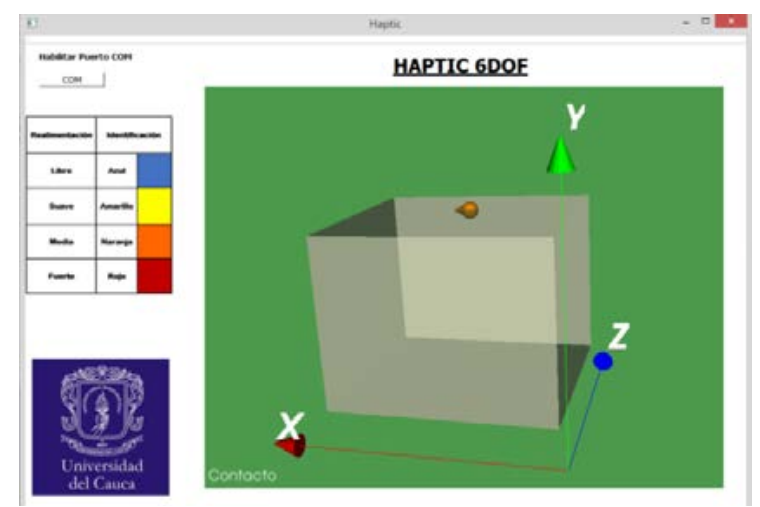

Figura 10. Cursor virtual tocando la pared superior del cubo (realimentación media)

Fuente: elaboración propia. 

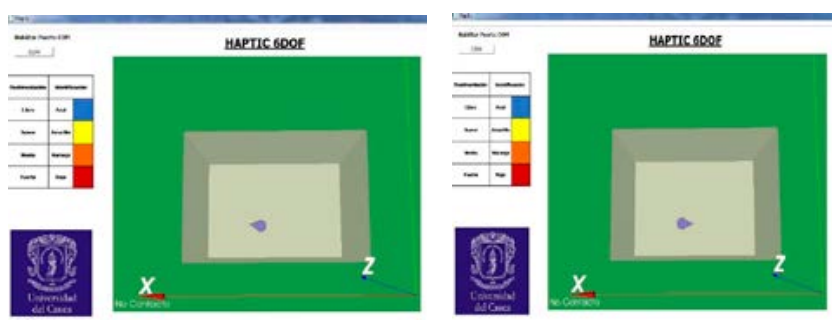

Figura 11. Variación de la orientación en $Z$ del cursor virtual

Fuente: elaboración propia.

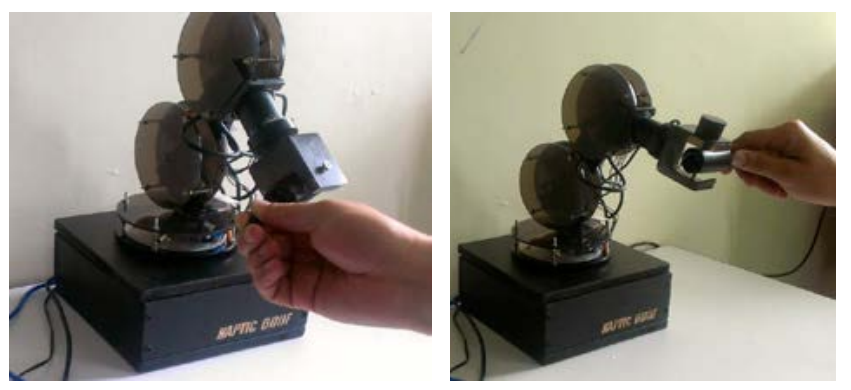

Figura 12. Movimiento de la interfaz mientras se varía la orientación en z del cursor virtual

Fuente: elaboración propia.

Para el caso de la figura 10 donde el cursor virtual toca una de las paredes del cubo, el usuario ha realizado una fuerza que equivale a una realimentación media de los actuadores de la interfaz, lo cual se manifiesta en el color naranja del cursor.

La interfaz fue evaluada utilizando veinte usuarios: diez estudiantes de pregrado en ingeniería, cuatro estudiantes de posgrado en ingeniería, cuatro docentes con conocimientos en robótica, y dos docentes en ingeniería sin conocimientos en robótica, todos los docentes de la Facultad de Ingeniería Electrónica de la Universidad del Cauca. A los usuarios se les pidió, inicialmente, que leyeran un breve manual de manejo de la interfaz, para realizar luego una serie de ejercicios con ella antes de contestar una encuesta. El $80 \%$ de los encuestados expresó su alta satisfacción respecto al manejo de la interfaz y a la sensación de realimentación que obtuvieron cuando el cursor tocaba los límites del cubo virtual. De otra parte, el $70 \%$ manifestó que el uso de la interfaz fue bastante sencillo. Como aspectos negativos se resalta el temor que tienen los usuarios al realizar mucha fuerza en las zonas límite del cubo. Aunque los engranajes utilizados son metálicos, se piensa utilizar electroimanes para una nueva versión de la interfaz, lo cual le daría mucha mayor robustez al dispositivo.

\section{CONCLUSIONES}

El presente artículo mostró el diseño y posterior construcción de una interfaz háptica de seis grados de libertad, denominada Haptic 6DOF. Se diseñó la interfaz siguiendo las características de un robot industrial de seis grados de libertad tipo Puma. Se obtuvieron los modelos matemáticos de la interfaz y se realizó su simulación en el ambiente Matlab Simulink. Esto permitió hallar los pares que van a los motores del dispositivo, lo cual sirvió de guía para la compra de los mismos. Se construyó la interfaz teniendo en cuenta que las tres primeras articulaciones permiten posicionar el cursor virtual en un espacio tridimensional, y las tres últimas proporcionan la orientación de dicho cursor.

Se ideó un sistema de realimentación que consiste en dos discos anclados por medio de resortes, los cuales proporcionan la realimentación virtual al usuario. Se construyó un software de manejo utilizando como motor gráfico la herramienta VTK, donde se tiene un cubo y, en el interior de este, un cursor virtual, movido por el usuario. Se planteó una realimentación en tres fases: suave, media, y fuerte, dependiendo de la penetración que realice el cursor virtual dentro de las paredes del cubo.

La interfaz fue probada por diversos usuarios, de los cuales, la mayaría de ellos manifestó su satisfacción respecto a la funcionalidad y a la sensación de realimentación. Futuros trabajos incluirán un sistema de realimentación proporcional a la fuerza que ejerce el usuario ( $y$ no en tres niveles fijos como se tiene actualmente), así como algoritmos de deformación para proporcionar mayor realismo a la interfaz gráfica. 


\section{REFERENCIAS}

Abate, A.; Guida, M.; Leoncini, P.; Nappi, M. y Ricciardi, S. (2009). A haptic-based approach to virtual training for aerospace industry. Journal of Visual Languages \& Computing, 20(5), 318-325. https:// doi.org/10.1016/j.jvlc.2009.07.003

Bueno, M.; Arteaga, M. y Candea, A. (2013). Modelado de sistemas de visión en 2D y 3D: un enfoque hacia el control de robots manipuladores. Tecnura, 17(37), 23-21.

Gómez, M. y Flórez, L. (2016). Modelado de objetos heterogéneos: una propuesta con base en funciones implícitas. Tecnura, 20(47), 15-27.

Hollis, R. (2010). Butterfly Haptics: A High-Tech StartUp. IEEE Robotics \& Automation Magazine, 17(4), 14-17. https://doi.org/10.1109/MRA.2010.938835

Khalil, W. y Kleinfinger J. (1986). A new geometric notation for open and closed loop robots. Proceedings IEEE Conference on Robotics and Automation, San Francisco, EE. UU. https://doi.org/10.1109/ ROBOT.1986.1087552

Khalil, W. y Creusot, D. (1997). Symoro+: A System for the Symbolic Modelling of Robots. Robotica, 15, 153-161. https://doi.org/10.1017/ S0263574797000180

Mosquera, V.; Vivas, A. y Rengifo C. (2011). Modelado y simulación de un robot para cirugía endoscópica transluminal. Revista Ingeniería Biomédica, 5(10), 44-50.

Ohnishi, K.; Shimono, T. y Natori, K. (2009). Haptics for medical applications. Artificial Life and Robotics, 13(2), 383-389. https://doi.org/10.1007/ s10015-008-0624-3
Salinas, S. y Vivas, A. (2009). Modelado, simulación y control del robot para cirugía laparoscópica LapBot. Revista Chilena de Ingeniería, 17(3), 317-328. https://doi.org/10.4067/S0718-33052009000300005

Salisbury, J. y Srinivasan, M. (1997). PHANToM-based haptic interaction with virtual graphics. IEEE Computer Graphics Applications, 17(5), 6-10. https:// doi.org/10.1109/MCG.1997.1626171

Tiwana, M.; Redmond, S. y Lovell, N. (2012). A review of tactile sensing technologies with applications in biomedical engineering. Sensors and Actuators A: Physical, 179, 17-31. https://doi.org/10.1016/j. sna.2012.02.051

Van der Linde, R.; Lammertse, P.; Frederiksen, E. y Ruiter, B. (2002). The hapticmaster, a new highperformance haptic interface. Edimburgo, Reino Unido: Proceedings of Eurohaptics'02.

Vander Poorten, E.; Demeester, E. y Lammertse, P. (2012). Haptic feedback for medical applications, a survey. Bremen, Alemania: Proceedings Actuator 2012.

Vaca, J.; Pe-a, C. y Vacca, H. (2015). Cinemática inversa de robot serial utilizando algoritmo genético basado en MCDS. Tecnura, 19(44), 33-45. https://doi. org/10.14483/udistrital.jour.tecnura.2015.2.a02

Vera, D. y Vivas, A. (2013). Ambiente virtual para el entrenamiento de cirugías laparoscópicas utilizando robots. Pan American Health Care Exchanges. Medellín, Colombia.

Vivas, A. (2010). Dise-o y control de robots industriales: teoría y práctica. Buenos Aires, Argentina: El Aleph.

Vivas; A.; Rengifo, C. y Mosquera V. (2011). Simulador quirúrgico para operaciones de laparoscopia utilizando interfaces hápticas. XIV Convención de Ingeniería Eléctrica. Santa Clara, Cuba.

\section{(C) $(1) \Theta \Theta$}

